NOTA CIENTÍFICA

\title{
Actividad larvicida de la toronja, Citrus paradisi (Rutaceae) sobre dos vectores del dengue
}

\section{Larvicidal activity of the grapefruit Citrus paradise (Rutaceae) on two vectors of dengue}

${ }^{1}$ Instituto Smithsonian de investigaciones Tropicales, Republica de Panamá.

${ }^{2}$ Departamento de química, Facultad de Ciencias naturales, Exactas y tecnología, Universidad de Panamá.

${ }^{3}$ Departamento de Entomología, Instituto Conmemorativo Gorgas de Investigaciones de la Salud, Republica de Panamá.

Instituto Smithsonian de Investigaciones Tropicales. Apartado 0843-00153, Panamá, Republica de Panamá.

E-Mail Jorge Morales: moralesj@si.edu

Presentado: $\quad$ 10/09/2006 Aceptado: $\quad 03 / 05 / 2007$

\section{Jorge Morales-Saldaña', Nélida Gómez', José Rovira², Manuel Abrahams ${ }^{3}$}

\section{Resumen}

La actividad larvicida del aceite esencial de la toronja (Citrus paradisi) fue evaluada contra los mosquitos Aedes aegypti y Aedes albopictus, los cuales representan vectores potenciales de la fiebre del dengue. $\mathrm{La} \mathrm{CL}_{50}$ del aceite esencial de la toronja fue de 47,3 ppm y 85,1 ppm para Ae. aegypti y Ae. albopictus, respectivamente. El análisis del aceite por GC-MS, mostró químicos conocidos como insecticidas los cuales podría explicar la acción tóxica sobre las larvas de los mosquitos evaluados. Esta actividad tóxica sobre Ae. aegypti fue mayor en comparación con otros aceites cítricos, lo que sugiere mayores instigaciones en esta línea y de esta manera poder recomendarlo como regulador de mosquitos a las diferentes campañas contra el Ae. aeygpti. En la literatura revisada no encontramos ninguna investigación donde haya sido evaluado aceites esenciales de cítricos contra larvas del mosquito Ae. albopictus.

Palabras Claves: Citrus paradisi, Actividad Larvicida, Aedes agypti, Aedes albopictus.

\section{Abstract}

Larvicidal activity of the grapefruit (Citrus paradisi) was tested against mosquitoes Aedes aegypti and Aedes albopictus, which represent potentials vectors of dengue fever. The $\mathrm{CL}_{50}$ of the essential oil of the grapefruit were 47,3 ppm and 85,1 ppm for Ae. aegypti and Ae. albopictus, respectively. The analysis of the oil by GC-MS, showed different components knowns as insecticides, which could explain the toxicity of this oil against mosquitoes. This toxic activity against Ae. aegytpi was higher in comparison whit another citrus oils, that suggest further researches in this line for be recommend in the different campaigns against the mosquito Ae. aegypti. In the literature revised we did not find research in where has been evaluated the essential oil of citrus against larvae of mosquito Ae. alboictus

Keywords: Citrus paradisi, Larvicidal Activity, Aedes agypti, Aedes albopictus.

\section{Introducción}

La Fiebre del Dengue (FD) y el Dengue hemorrágico (DH) representan enfermedades virales de mayor importancia epidemiológica en áreas tropicales y subtropicales. Actualmente cada año alrededor de 50 a 100 millones de casos de fiebre del dengue ocurre en todo el mundo, con aproximadamente 250000 a 500000 casos de dengue hemorrágico (Rigau-Perez et al.,1998) Debido a esto existe gran esfuerzo científico enfocado en encontrar la vacuna contra esta enfermedad (Chaturvedi et al., 2005). Pero hasta ahora, la mejor forma de reducir su incidencia es controlando a sus vectores (Severson et al., 2004), principalmente atacando sus sitios de cría (Briegel, 2003; Carvalho et al., 2003). No obstante, el mal uso de los químicos empleados para esta labor han afectado negativamente al medio ambiente y a los seres humanos (Silva et al., 2004; Thomas et al., 2000; Thomas et al., 2004). Además, los mosquitos sobre los cuales se aplican estos químicos han desarrollado altos niveles de resistencia a los mismos (Brown, 1986); lo que se traduce en grandes pérdidas económicas.

Actualmente existen nuevas y mejores alternativas para el control de mosquitos, como por ejemplo: liberación de mosquitos genéticamente modificados y químicos provenientes de plantas o químicos naturales (Sukumar et al., 1991; Scott et al., 2002) figuran entre los mejores. Sin embargo, esta última alternativa (químicos naturales), relativamente simple, podría ser desarrollada a mayor brevedad, y con menor costo que las otras. Además, se ha demostrado su eficacia en comparación con químicos convencionales y una relativa menor toxicidad en los seres humanos y el medio ambiente (Sukumar et al., 1991; Bassolé et al., 2003).

Dentro de los grupos de plantas que han resultado positivas como insecticida están los cítricos (Calvacanti et al., 2004; Dakhil et al., 1999; Ezeonu et al., 2001; Jayaprakasha et al., 1997; Mwaiko, 1992; Mwaiko et al., 1994; Su et al., 1972). Entre los cuales se encuentra Citrus paradisi como uno de los mejores candidatos para ser un insecticida idóneo (Su et al., 1972); a pesar de esto, solo ha sido evaluada como larvicida de una especie de mosquito (Culex pipiens) (Dakhil et al. 1999), dejando en duda su acción larvicida sobre otras especies de mosquitos. De aquí nace el propósito de este trabajo, evaluar la actividad larvicida del aceite esencial de la toronja sobre dos mosquitos vectores del dengue, Aedes aegypti y Aedes albopictus. Además, brindar un perfil químico del aceite esencial de la toronja utilizado.

\section{Extracción y análisis del aceite esencial de la toronja}

La toronja madura fue comprada en un mercado local de la provincia de Colón, Republica de Panamá. El aceite de toronja fue obtenido a partir de $500 \mathrm{~g}$ de la cáscara o epicarpio según el método de Su et al. (1972), el cual también señala la manera adecuada con que se debe limpiar y cortar la cáscara antes de la extracción. Una vez extraído el aceite, fue diluido en $1 \mathrm{ml} \mathrm{de}$ etanol y guardado en refrigeración hasta su posterior uso. El 
aceite de la toronja fue analizado bajo GC Agilent $6890 \mathrm{~N}$ con detector selectivo de masa (MSD) Agilent 5973 de columna capilar HP-5MS con diámetro interno de $0,25 \mathrm{~mm}$, longitud de $30 \mathrm{~m}$ y $0,25 i ̀ m$ de espesor de película. Se programo el horno a temperatura inicial de $70{ }^{\circ} \mathrm{C}$ por 1 minuto, luego se elevó la temperatura hasta a $250{ }^{\circ} \mathrm{C}$ a razón de $30^{\circ} \mathrm{C} / \mathrm{min}$. El Helio fue utilizado como gas de arrastre a $0,9 \mathrm{ml} / \mathrm{min}$; con temperatura de inyección $250^{\circ} \mathrm{C}$. El tiempo de corrida total fue de $13 \mathrm{~min}$. Para la identificación de los componentes del aceite se comparó de forma computarizada los espectros obtenido del aceite con los de la librería digital Wiley (séptima edición).

\section{Crianza de los mosquitos Ae. aegyptiy Ae. albopictus}

Fue utilizada la cepa "Puerto armuelles" de Ae. aegypti y "Aedes albopictus" de Ae. albopictus ambas establecidas en el año 2003 dentro de la Sección de Entomología del Instituto Conmemorativo Gorgas de Investigaciones de la Salud (ICGES). Los mosquitos fueron criados de manera separada según la metodología utilizada en esta sección, la cual consiste en colocar 600 huevos de mosquitos dentro de una bandeja $(30 \times 15 \times 5 \mathrm{~cm})$ con 2 litros de agua y alimentarlas diariamente con $0,45 \mathrm{~g}$ de levadura. La temperatura y la humedad relativa del periodo de cría fueron de $27 \pm 2{ }^{\circ} \mathrm{C}$ y $85 \pm 3 \%$.

\section{Bioensayos}

Una vez llegado el cuarto estadio larval, grupos de 20 larvas fueron colocados en envases plásticos $(\approx 150 \mathrm{ml}$ de capacidad) con $100 \mathrm{ml}$ de agua ( $\mathrm{pH} 6,9)$ más la proporción de aceite de toronja necesaria para obtener las siguientes concentraciones: 0 (con $1 \mathrm{ml}$ de etanol), 30, 60, 90, 120, 150 ppm. Estas concentraciones fueron establecidas después de múltiples ensayos preliminares. Cada concentración fue replicada 4 veces, para cada especie de mosquito. La mortalidad larval fue registrada a las 24 horas de haberse expuesto las larvas a las diferentes concentraciones del aceite. Durante este tiempo no se le ofreció alimento. El criterio de mortalidad larval fue al no recibir ningún tipo de respuesta después de ser tocadas con un alfiler en la región del sifón o la región cervical. Las condiciones de laboratorio fueron las mismas del periodo de crianza. Se calcularon las concentración letales al $50 \%\left(\mathrm{CL}_{50}\right)$ y $90 \%\left(\mathrm{CL}_{90}\right)$ bajo el programa EPA Probit analysis versión 1,5 .

\section{Resultados y Discusión}

La tabla 1 muestra la actividad larvicida del aceite esencial de la toronja sobre las larvas de Ae. aegypti y Ae. albopictus. Como puede ser observado la $\mathrm{CL}_{50}$ para el $A e$ agypti y para $A$ e. albopictus fue de 47,3 ppm y 98,9 ppm respectivamente. El 100\% de mortalidad larval fue registrada únicamente en Ae. aegypti en concentraciones de $150 \mathrm{ppm}$ del aceite de toronja, mientras

Tabla 1. Actividad larvicida del aceite esencial de la toronja (Citrus paradisi) contra los mosquitos Aedes aegypti y Aedes albopictus

\begin{tabular}{lccc}
\hline Aedes & LC $_{50}$ ppm & LC $_{90}$ ppm & $\begin{array}{c}\text { Heterogeneity } \\
\mathbf{X}^{2}(\mathbf{d f})^{*}\end{array}$ \\
\hline aegypti & 47,3 & 98,9 & \\
& $(42,1-52,2)^{* *}$ & $(88,6-113,6)^{* *}$ & $4,140(3)^{*}$ \\
albopictus & 85.9 & 147.5 & \\
& $(78,7-92,7)^{* *}$ & $(131,4-160,3)^{* *}$ & $3,943(3)^{*}$ \\
\hline
\end{tabular}

Grados de libertad, **Limites de confianza.
Tabla 2. Principales componentes del aceite esencial de la toronja (Citrus paradisi)

\begin{tabular}{lcc}
\hline Componentes & Tiempo de retención & $\begin{array}{c}\text { Porcentaje de } \\
\text { composición }\end{array}$ \\
\hline$\alpha$-Pinene & 2,70 & 0,60 \\
Sabinene & 2,99 & 1,04 \\
$\beta$-Pinene & 3,08 & 3,27 \\
Octanal & 3,17 & 2,13 \\
Ocimene & 3,54 & 53,04 \\
Delta3-carene & 3,58 & 1,08 \\
Limonene & 3,79 & 0,41 \\
Linalool oxide & 3,95 & 0,77 \\
Linalool L & 4,02 & 2,12 \\
Nonanal & 4,05 & 0,58 \\
Trans-Limonene & 4,25 & 0,09 \\
Citronella & 4,50 & 0,38 \\
Decanal & 4,99 & 2,01 \\
$\beta$-Citronellol & 5,18 & 0,39 \\
Nerol & 5,21 & 0,48 \\
Z-Citral & 5,34 & 0,82 \\
Geraniol & 5,43 & 0,08 \\
1-Decanol & 5,58 & 0,65 \\
$\alpha$-Copaene & 6,65 & 0,47 \\
$\beta$-Cubebene & 6,77 & 0,44 \\
Dodecanal & 6,82 & 0,10 \\
Caryophyllene & 7,08 & 0,77 \\
Trans- $\beta$-Farnesene & 7,24 & 0,08 \\
$\alpha$-Humelene & 7,36 & 0,15 \\
Germacrene-D & 7,59 & 0,37 \\
Bicyclogermacrene & 7,72 & 0,20 \\
Delta-Cadinene & 7,86 & 0,17 \\
Nerolidol & 8,15 & 0,11 \\
Octadecanal & 8,51 & 0,13 \\
Ledol & 9,00 & 0,13 \\
Tetrinensal & 9,23 & 0,13 \\
Methyl Palmitate & 10,02 & 0,07 \\
\hline & 10,78 & 0,18 \\
\hline
\end{tabular}

que Ae. albopictus presentó mortalidad de $92 \%$ en la misma concentración. En la concentración de 30 ppm, Ae. aegypti y Ae. albopictus presentaron mortalidades larvales de $22 \%$ y $12 \%$, respectivamente. Ambas especies se mostraron susceptibles al aceite esencial de la toronja; sin embargo este aceite resulto ser 2,1 veces mas toxico para el mosquito Ae aegypti que para Ae. albopictus. Esta pequeña diferencia quizás se deba a distintas formas de metabolizar este tipo de sustancias tóxicas. Se necesitaran más estudios para poder comprobar esta hipótesis.

Dakhil et al. (1999), reportaron actividad toxica de la toronja sobre las larvas del Culex pipiens. Al igual que ellos, nosotros demostramos actividad toxica del aceite esencial de toronja sobre los mosquitos, Ae. aegypti y Ae. albopictus, los cuales constituyen vectores potenciales del virus del dengue.

Esta es la primera evaluación de la toxicidad del aceite esencial de la toronja sobre larvas de Ae. aegypti. Anteriormente la propiedad larvicida de otros aceites cítricos como Citrus sinensis y Citrus limonia habían sido investigados sobre Ae. aegypti; sin embargo, la actividad larvicida de estos aceites, $\mathrm{CL}_{50}: 538 \mathrm{ppm}$ y $\mathrm{CL}_{50}: 519$, respectivamente (Calvacanti et al., 2004), fue menor en comparación con la reportada en la presente investigación, $\mathrm{CL}_{50}: 47,3$ ppm; es decir 11 veces más toxica para las larvas del mosquito Ae. aegypti. En la literatura revisada no se encontró 
ninguna investigación acerca de aceites esenciales cítricos como larvicida del mosquito Ae. albopictus.

La producción de aceite esencial fue de 1,2\% de cáscara de toronja. Los compuestos obtenidos en el análisis químico del aceite son mostrados en la tabla 2. Se logró la obtención de 33 compuestos de los cuales ocimene $\beta$ (53,04\%), $\beta$ - pinene $(3,27 \%)$, linalool $(2,12 \%)$, octanal $(2,13 \%)$ y decanal $(2,01 \%)$ se encontraban en mayor porcentaje de composición del aceite. Los compuestos con pequeñas cantidades fueron limonene $(0,41 \%)$ $y$, genariol y trans_ $\beta$ - farnesene ambos con $0,08 \%$.

Con el análisis químico del aceite de toronja se pudo comprobar con alta probabilidad la ausencia de insecticidas convencionales que pudieran estar en la cáscara de la toronja y la presencia de químicos naturales con propiedades insecticidas como por ejemplo: linalool, ocimene, myrcene, $\alpha$-pinene, sabinene, limonene y $\beta$-carophyllene (Jaenson et al., 2006). Que pudieran explicar la toxicidad de este aceite sobre las larvas de mosquitos.

El aceite esencial de la toronja ha demostrado ser un buen candidato como regulador de mosquito; no obstante, es necesario más investigaciones acerca de su especificidad, residualdad y de su comportamiento en el medio ambiente antes de ser recomendado en las campañas contra el dengue.

\section{Agradecimientos}

A JD, Gina Mow y al personal de la Sección de Entomología del instituto Gorgas por su apoyo en el laboratorio y en la revisión del manuscrito.

\section{Literatura Citada}

Bassolé I., W. Guelbeogo, R. Nébié, C. Constantini, N. Sagnon, Z. Kabore \& S. Traeoré. 2003. Ovicidal and larvicidal activity against Aedes aegypti and Anopheles gambiae complex mosquitoes of essential oila extrated from three spontaneuos plants of Burkina Faso. Parassitologia 45: 23- 26.

Briegel H. 2003. Physiological bases of mosquito ecology. J. Vec. Ecol. 28 (1): 1-11.

Brown A. 1986. Insecticide resistance in mosquitoes: A pragmatic review. J. Am. Mosq. Control Assoc. 2: 123-140.

Calvacanti E., S. Morais, M. Lima \& E. Santana 2004. Larvicidal activity of essential oils from Brazilian plants against Aedes aegypti. Mem. Inst. Oswaldo Cruz. 99(5): 541-544.

Carvalho A. U. F., V. M. M. Melo, A. A. Craveiro, et al. 2003. Larvicidal activity of the essential oil from Lippia sidoides cham. against Aedes aegypti linn. Mem. Inst. Oswaldo Cruz. 98(4): 569-571.
Chaturvadi U., R. Shrisvastava \& R..Nagar 2005. Dengue vaccines: Problems and Prospects.Indian J. Med. Res. 121: 639-652.

Dakhil M \& T. Morsy. 1999. The larvicidal activities of the peel oils of three citrus fruits against Culex pipiens. J. Egypt. Soc. Parasitol. 29 (2): 347-352.

Ezeonu F., G. Chidume \& S. Udedi. 2001. Insecticidal properties of volatile extracs of orange peels. Bioresource Technol. 76: 273-274.

Jaenson T., K. Palsson \& A. Borg-Karlson. 2006. Evaluation of extracts and oils of mosquito (Diptera:Culicidae)repellent plants from Sweeden and Guinea-Bissau. J. Med. Entomol. 43(1): 113-119.

Jayaprakasha R., R. Singh, J. Pereira \& K. Sakariah. 1997. Limonoids from Citrus reticulata and their moult inhibiting activity mosquito Culex quinquefasciatus larvae. Phytochemistry 44: 843-846.

Rigau-Perez J., G. Clark, D. Gubler, P. Reiter, E. Sanders \& A. Vornden. 1998. Dengue and dengue haermorrhagic fever. Lancet. 352(19): 971-977.

Mwaiko G. 1992. Citrus peels oil extracts as mosquito larvae insecticides. E. Afr. Med. J. 69(4): 223-226.

Mwaiko G. \& Z.Savaeli. 1994. Lemon peel oil extract as mosquito larvicide. E. Afr. Med. J. 71 (12): 797-799.

Scott T., W. Takken, B. Knols \& C. Boete. 2002. The Ecology of modified mosquitoes. Science. 298(4):117-119.

Severson D., D. Knudson, M. Soares \& B. Loftus 2004. Aedes aegypti genomics. Insect Mol. Biol. 34: 715-721.

Silva H., I. Silva, R. Santos, E. Filho \& C. Elias. 2004. Atividade larvicida de taninos isolados de Mangonea Pubescens St. Hil(Sapindaceae) sobre Aedes aegypti (Diptera, Culicidae) Rev. Soc. Bras. Med. Trop.35(5): 1438-1441.

Sukumar K., M. Perich \& L. Boobar. 1991. Botanical derivates in mosquito control: A review. J. Am. Mosq. Control. Assoc. 7(2): 211-237.

Su H., R. Speirs \& Mahany. 1972. Toxicity of citrus oils to several stored-product insects: Laboratory evaluation. J. Econ. Entomol. 65(6): 1438-1441.

Thomas T., S. Sharma, A. Prakash \& B. Sharma. 2004.Insecticidal properties of essential oil of Cannabis sativa Linn. Against mosquito larvae. Entomon 25(1):21-24.

Thomas T., S. Rao \& S. Lal. 2004. Mosquito larvicidal properties of essential oil of an indigenous plant, Ipomoea cairica Linn. Jpn. J. Infect Dis. 57:176-177. 


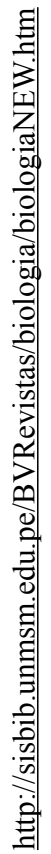

